\title{
Headache yesterday in Karnataka state, India: prevalence, impact and cost
}

Timothy J. Steiner ${ }^{1,2^{*}}$, Girish N. Rao ${ }^{3}$, Girish B. Kulkarni ${ }^{4}$, Gopalkrishna Gururaj ${ }^{3}$ and Lars J. Stovner ${ }^{1,5}$

\begin{abstract}
Background: The Global Campaign against Headache has pioneered evaluation of the prevalence and impact of headache on the preceding day ("headache yesterday") as a new approach to the estimation of headacheattributed burden, avoiding recall error. We report its application in Karnataka State, southern India.

Methods: In a door-to-door survey, biologically unrelated adults (aged 18-65 years) were randomly sampled from urban and rural areas in and around Bengaluru and interviewed by trained researchers using a validated, structured questionnaire. Enquiry into headache applied ICHD-II diagnostic criteria and included questions about headache on the day preceding the interview (headache yesterday [HY]).

Results: There were 2329 participants (participation proportion $92.6 \%$; males 1141 [49.0\%], females 1188 [51.0\%]; mean age 38.0 [ \pm 12.7 ] years; 1103 [47.4\%] from rural areas, 1226 [52.6\%] urban). HY was reported by 138 participants (males 33 [2.9\%], females 105 [8.8\%]): the 1-day prevalence of headache was $5.9 \%$. Mean duration of HY was $7.0 \pm 8.5 \mathrm{~h}$, so that $1.7 \%$ of the population (5.9\%* 7.0/24), on average, had headache at any moment in time yesterday. Mean intensity on a scale of $1-3$ was 2.0 [ \pm 0.8 ]. Lost productivity due to HY was reported by $83.3 \%$ of participants with HY: $37.7 \%$ able to do less than half of what they had planned and $13.0 \%$ able to do nothing. Productivity loss at population level (being the productivity loss within the entire adult population, every single day, attributable to headache) was $3.0 \%$.
\end{abstract}

Conclusions: This method of enquiry, free from recall error, confirmed a very high level of headache-attributed burden in Karnataka: previous estimates based on 3-month recall may even have been too low. Until another study is done in the country, these are the best data for all India. They demonstrate need for action nationwide to mitigate this burden, and correct action will ultimately almost certainly be cost-saving.

Keywords: Headache yesterday, Migraine, Tension-type headache, Medication-overuse headache, Burden, Cost, Health policy, India, Global Campaign against Headache

\section{Background}

The very heavy disability burden attributable to headache disorders is becoming increasingly clear $[1,2]$. The Global Burden of Disease Study 2010 (GBD2010) ranked tension-type headache (TTH) and migraine as the second and third most common diseases worldwide [3], but many of the data included in this world survey derived from heterogeneous studies performed with varying methods over a period of nearly 30 years. In all regions except the Far East, more recent studies-of migraine in

\footnotetext{
* Correspondence: t.steiner@imperial.ac.uk

'Department of Neuroscience, Norwegian University of Science and Technology (NTNU), Edvard Griegs Gate, Trondheim NO-7491, Norway

${ }^{2}$ Division of Brain Sciences, Imperial College London, London, UK

Full list of author information is available at the end of the article
}

particular-have generally yielded higher prevalence estimates than the global mean estimates of GBD2010 [3] or GBD2013 [1]. This has been particularly apparent in low- and lower-middle-income countries: Georgia [4], India [5], Nepal [6], Zambia [7], Ethiopia (unpublished) and Pakistan (unpublished). In part this is explained by the careful methodology of recent studies $[8,9]$. To a greater extent it may be because many earlier published studies excluded probable migraine: many reports are silent on how ICHD diagnostic criteria were applied with respect to definite and probable migraine; some explicitly excluded the latter, but a larger number may have done so without making this clear. 
The expectation, as the Global Campaign against Headache $[10,11]$ continues its drive to conduct population-based studies in those parts of the world where data are currently lacking-mostly in low- and lower-middle-income countries, is that global mean prevalence estimates will continue to rise. With them, estimates of burden will rise also, as they have done through the iterative GBD studies since 2000 [2]. These estimates are the basis of needs assessments informing health policy and allocation of health-care resources.

When disease burdens are large and the call upon resources is substantial, estimates need to be as accurate as possible. The methodology for measuring headacheattributed burden is a relatively new and still developing science $[8,9]$ : in the last 30 years, many populationbased studies of headache have assessed prevalence only. It is a field of innovation to which Lifting The Burden (LTB) $[12,13]$ has actively contributed in leading the Global Campaign against Headache. The components of headache-attributed burden are wide-ranging, and some are challenging to measure [9]. Symptom burden can be measured in terms of frequency, intensity and duration. The GBD studies use frequency and duration to estimate the proportion of time spent with symptoms, and apply a disability weight to this proportion of time to calculate the associated health loss [3]. For socioeconomic impact, the Headache-Attributed Lost Time (HALT) Index [14] enquires into effect of headache on productivity. In its original form (HALT-90), it relied upon a respondent's recall over the 3 months prior to the interview, that period of enquiry being intended to gather a representative view of each participant's lost productive time. This approach has its uses, but is subject to the limitations, errors and possible biases of recall over such a long period.

LTB has pioneered an alternative approach to avoid the problems associated with recall error, which is enquiry into headache occurring on the day before the interview ("headache yesterday"; HY) [8]. Recollection of $\mathrm{HY}$, its symptoms and whatever impact they may have had, is likely to be highly reliable. While what happened yesterday is not representative, or therefore indicative, of an individual's headache-attributed burden over longer periods of time, at population level it is. This approach does, however, require a large number of participants, since far fewer people have headache in 1 day than in 3 months.

A question subset concerning HY [9] has been included in several recent large epidemiological studies supported by LTB within the Global Campaign against Headache: in China [15], Russia [16], countries of the Eurolight project [17] and several others not yet published. Here we report the analysis of HY in Karnataka State, southern India, and make comparisons with estimates based on 3 months' recall [18]. We apply the analysis in making an estimate of socioeconomic burden attributable to headache disorders in Karnataka State, and consider extrapolating this to all India.

\section{Methods}

The methods of the study have been published in detail previously [19] and are described only briefly here.

\section{Study design and sampling}

This was a cross-sectional survey, conducted during May to November 2009. It sampled urban and rural areas in and around Bengaluru: Kempegowdanagara, an urban administrative ward in the city of Bengaluru, and Uyamballi and Doddaaladahalli, two large villages located $75-80 \mathrm{Km}$ from Bengaluru. Trained interviewers selected households randomly through multistage cluster sampling. They called at each household unannounced, listed all adult members (aged 18-65 years), randomly selected one and interviewed that person.

\section{Enquiry and diagnosis}

The survey instrument was a cultural adaptation of LTB's structured HARDSHIP questionnaire [9], translated into the local language (Kannada) in accordance with LTB's translation protocol for hybrid documents [20], and validated [19]. It began with demographic enquiry. The headache screening question ("Have you had headache during the last year?") and diagnostic questions based on ICHD-II [21] were followed, for those reporting headache, by multiple question sets enquiring into various aspects of burden, including the HALT questionnaire [14]. Participants reporting more than one headache type were asked to focus only on the one that was subjectively the most bothersome for purposes of diagnosis and burden attribution.

Diagnoses were made algorithmically. Participants with headache on $\geq 15$ days/month were first identified; among these, any with medication overuse were diagnosed as probable $\mathrm{MOH}(\mathrm{pMOH})$, the remainder as "other headache on $\geq 15$ days/month". The algorithm then applied ICHD-II criteria to all other cases in hierarchical sequence: first for definite migraine, then for definite TTH, then for probable migraine and finally for probable TTH. Remaining cases were considered unclassifiable. In the analysis, definite migraine and probable migraine were grouped as migraine, and definite TTH and probable TTH as TTH.

Questions on HY (in response to "Did you have headache yesterday?") included duration of HY (in hours up to 24), intensity of HY (reported by participants on a three-point categorical score ["not bad", "quite bad" and "very bad"]) and usage of acute medications. Lost productivity because of HY was reported as what had been 
done yesterday considered with regard to what had been planned or expected to be done: "everything", "more than half ", "less than half" or "nothing". The analysis of HALT offsets those who had done less than half against those who had done more than half, counting the former as doing nothing and the latter as doing everything $[9,14]$.

\section{Statistics and analyses}

Data were entered into a secure database and statistical analyses were performed using EPI INFO [22] and SPSS version 15.

We used proportions (\%) to report prevalences, with $95 \%$ confidence intervals (CIs) when appropriate. We used means, with standard deviations (SDs) and/or standard errors (SEs), and medians to summarise the distributions of continuous variables. Headache intensity responses "not bad", "quite bad" and "very bad" were equated to "mild", "moderate" and "severe" and converted to a numerical rating scale $1-3$, which we treated as continuous data. We used Fisher's exact test for significance of differences. We set the level of significance at $p<0.05$.

We calculated the predicted 1-day prevalence of any headache and of headache types from the 1-year prevalence and estimated mean frequency (F) in days/year (extrapolated from the last 3 months) using the formula:

$$
1 \text {-day prevalence }=1 \text {-year prevalence } * \mathrm{~F} / 365
$$

We calculated total lost productivity as a proportion (\%), the numerator being the sum of those reporting nothing or less than half done and the denominator the number $(\mathrm{N})$ who reported $\mathrm{HY}$.

\section{Results}

There were 2329 participants (1141 [49.0\%] male, 1188 [51.0 \%] female; mean age 38.0 [ \pm 12.7$]$ years; 1103 [47.4\%] from rural areas, 1226 [52.6 \%] urban). The participation proportion was $92.6 \%$ (eligible population $n=$ 2514). The distributions of gender, age and habitation in the participating sample have been described in detail previously and were comparable to those of the population of Karnataka [19]. As reported previously, the 1year prevalence of any headache was $63.9 \%$; the agestandardised 1-year prevalence of migraine was $25.2 \%$ and of TTH was $35.1 \%$; the age-standardised prevalence of all causes of headache on $\geq 15$ days/month was $3.0 \%$ and of $\mathrm{pMOH}$ was $1.2 \%$ [5].

\section{Headache yesterday}

HY was reported by 138 participants, representing a 1-day prevalence of headache of $5.9 \%$. The predicted 1-day prevalence of any headache calculated from its 1-year prevalence of $63.9 \%$ and estimated mean frequency of 32.0 days/year [5] was $5.6 \%$, a very close match.

HY was reported by over three times as many females $(8.8 \%)$ as males (2.9 \%) (Table 1). Reporting of HY varied with age, but with wide and overlapping confidence intervals. Almost three quarters of HY (73.9 \%) was reported by rural participants, despite that they made up less than half $(47.4 \%)$ of the participating sample. These data are also shown in Table 1.

HY was reported to be typical of their usual most bothersome (diagnosed) headache by only 95 (68.8 \%) of those with HY. This limited the inferences we could draw about HY diagnostically. Among these 95 only, we could make reasonable assumptions as to diagnosis; on these, we based calculations of predicted 1-day prevalences by headache type (Table 2). There was an exact match for migraine and a close match for $\mathrm{pMOH}$; TTH and other headache on $\geq 15$ days/month were underreported yesterday, and may have been preferentially among the cases of $\mathrm{HY}$ not considered typical of the usual most bothersome headache. For all HY, as noted above, there was a good match.

Mean duration of HY was $7.0 \pm 8.5 \mathrm{~h}$. From this we calculated that $1.7 \%$ of the population $(5.9 \% * 7.0 / 24)$, on average, had headache at any moment in time yesterday. The median was $3 \mathrm{~h}$; therefore HY was quite shortlasting in more than half of cases. If this reflected usage of acute medications, these were taken by $46.3 \%$ of participants with HY-that is, $2.5 \%$ of the total survey sample. By implication this meant that, on any day, $2.5 \%$ of the adult population were using acute medication for headache. A further $36.9 \%$ used local applications of various sorts, including $4.3 \%$ who used both medication and local applications. Headache intensity was severe in $29.7 \%$, moderate in $46.4 \%$ and mild in $23.9 \%$. Mean intensity on a scale of $1-3$ was therefore $2.0[ \pm 0.8]$ (SE: 0.07).

Lost productivity due to HY was reported to some degree by $83.3 \%$ of participants with HY: $37.7 \%$ had been able to do less than half of what they had planned and $13.0 \%$ could do nothing. Applying the concept behind the analysis of HALT (see Methods [14]), we found that HY cost those affected by HY half (50.7\%) of their productivity yesterday. We looked at this by diagnosis in those for whom we had diagnostic information (Table 3). This analysis indicated that migraine cost much more lost productivity than TTH ( $p=0.048$ [Fisher's exact, 2-tailed]), while $\mathrm{pMOH}$ eclipsed even migraine (but with small numbers, $p=0.75$ ). To calculate the productivity loss due to $\mathrm{HY}$ at population level we multiplied $50.7 \%$ by the overall prevalence of $\mathrm{HY}$ of $5.9 \%$; this gave an estimate of $3.0 \%$ as the productivity loss within the entire adult population, every single day, attributable to headache. 
Table 1 Characteristics of participants reporting headache yesterday $(H Y)$ and population prevalence of $H Y$ by group ( $N=2329)$

\begin{tabular}{|c|c|c|c|}
\hline Characteristic & $\begin{array}{l}\text { Reported cases of HY } \\
\text { in defined group } \\
\mathrm{n}(\%)\end{array}$ & $\mathrm{N}$ in defined group & $\begin{array}{l}\text { Population prevalence } \\
\text { of HY in defined group } \\
\text { (\%) }[95 \% \mathrm{Cl}]\end{array}$ \\
\hline All cases of HY & $138(100.0)$ & 2329 & $5.9[5.0-7.0]$ \\
\hline \multicolumn{4}{|l|}{ Gender } \\
\hline Male & $33(24.0)$ & 1141 & $2.9[2.1-4.0]$ \\
\hline Female & $105(76.0)$ & 1188 & $8.8[7.4-10.6]$ \\
\hline \multicolumn{4}{|l|}{ Age (years) } \\
\hline$<25$ & $18(13.0)$ & 353 & $5.1[3.3-7.9]$ \\
\hline 25 to 34 & $35(25.4)$ & 627 & $5.6[4.0-7.7]$ \\
\hline 35 to 44 & $41(29.7)$ & 603 & $6.8[5.1-9.1]$ \\
\hline 45 to 54 & $23(16.7)$ & 409 & $5.6[3.8-8.3]$ \\
\hline 55 to 65 & $21(15.2)$ & 337 & $6.2[4.1-9.3]$ \\
\hline \multicolumn{4}{|l|}{ Habitat } \\
\hline Rural & $102(73.9)$ & 1103 & $9.3[7.7-11.1]$ \\
\hline Urban & $36(26.1)$ & 1226 & $2.9[2.1-4.0]$ \\
\hline
\end{tabular}

\section{Discussion}

The principal finding was a 1-day prevalence of headache of $5.9 \%$, with the implication that this proportion of the adult population (one in 17) had headache on each and every day. Mean duration of HY was $7.0 \pm$ $8.5 \mathrm{~h}$, so that $1.7 \%$ of the population $(5.9 \% * 7.0 / 24)$, on average, had headache at any moment in time yesterday (and, again by implication, at any moment on any day). Mean intensity on a scale of 1-3 was 2.0. Lost productivity was reported by most $(83.3 \%)$ participants with HY, of whom $37.7 \%$ could do less than half of what they had planned and $13.0 \%$ could do nothing. Productivity loss due to $\mathrm{HY}$ at population level (being the productivity loss within the entire adult population, every single day, attributable to headache) was $3.0 \%$.

The large difference in prevalence of HY between rural (9.3\%) and urban populations (2.9) was in part explained by the higher rural 1-year prevalence of headache (71.2\% versus $57.3 \%$ ), thought to be due to adverse rural socioeconomic conditions (diet, stress and relative poverty) [5]. In addition, the poor rural availability and utilization of health-care facilities were likely to contribute to more frequent headache and therefore more HY. Probable $\mathrm{MOH}$ was more prevalent in rural (1.5\%) than in urban areas (0.9\%) [5]; because of its high frequency, this disorder is a major contributor to HY despite its low prevalence.

It is a limitation of enquiry into HY that diagnosis cannot reliably be based on a single headache episode; unless a respondent is able to assert that HY was typical of a recurrent headache that has been diagnosed, its nature will not be known. In this survey, only two thirds could do this, with the result that our inferences on headache types, as opposed to all headache, are unavoidably constrained. From a public-health perspective this may not be of great importance; the key messages here emphasise the ubiquity of headache, its frequency, its symptom

Table 2 Prevalence of headache yesterday according to diagnosis (when recognized as most bothersome headache), and comparison with predicted 1-day prevalence based on reported frequency and 1-year prevalence

\begin{tabular}{|c|c|c|c|c|c|}
\hline \multirow[t]{2}{*}{ Headache type } & \multicolumn{2}{|c|}{$\begin{array}{l}\text { Headache yesterday: } \\
\text { with diagnosis }(N=95) \text { or all cases }(N=138)\end{array}$} & \multicolumn{3}{|c|}{ Predicted 1-day prevalence } \\
\hline & $\mathrm{n}(\%)$ & $\begin{array}{l}\text { Observed 1-day } \\
\text { prevalence }(\%)(N=2329)\end{array}$ & $\begin{array}{l}\text {-year } \\
\text { prevalence }{ }^{a}(\%)\end{array}$ & $\begin{array}{l}\text { Mean headache } \\
\text { days/year }\end{array}$ & $\begin{array}{l}\text { Calculated 1-day } \\
\text { prevalence (\%) }\end{array}$ \\
\hline Migraine & $44 / 95(46.3 \%)$ & 1.9 & 25.2 & $28^{b}$ & 1.9 \\
\hline Tension-type headache & 23/95 (24.2\%) & 1.0 & 35.1 & $17^{\mathrm{b}}$ & 1.6 \\
\hline Probable medication-overuse headache & 13/95 (13.7 \%) & 0.6 & 1.2 & $226^{b}$ & 0.7 \\
\hline Other headache on $\geq 15$ days/month & 15/95 (15.8\%) & 0.6 & 1.8 & $259^{b}$ & 1.3 \\
\hline Unknown headache yesterday & $43 / 138$ & 1.8 & - & - & - \\
\hline All headache yesterday & 138 & 5.9 & 63.9 & $32.0^{\mathrm{a}}$ & $5.6^{\mathrm{a}}$ \\
\hline
\end{tabular}

${ }^{\mathrm{a}}$ From reference [5]

${ }^{\mathrm{b}}$ From reference [18] 
Table 3 Lost productivity because of headache yesterday according to diagnosis (when recognized as most bothersome headache)

\begin{tabular}{|c|c|c|c|c|c|}
\hline \multirow[t]{2}{*}{ Amount of lost productivity } & \multicolumn{4}{|c|}{ Headache yesterday with diagnosis $(N=95)$} & \multirow{2}{*}{$\begin{array}{l}\text { Any } \\
\text { headache } \\
\text { yesterday } \\
(N=138)\end{array}$} \\
\hline & Migraine & $\begin{array}{l}\text { Tension-type } \\
\text { headache }\end{array}$ & Probable $\mathrm{MOH}$ & $\begin{array}{l}\text { Other headache } \\
\text { on } \geq 15 \text { days/month }\end{array}$ & \\
\hline $\mathrm{N}$ reporting headache yesterday $(X)$ & 44 & 23 & 13 & 15 & 138 \\
\hline Could do nothing at all (n) (Y) & 6 & 1 & 2 & 2 & 18 \\
\hline Could do less than half (n) (Z) & 20 & 6 & 7 & 3 & 52 \\
\hline $\begin{array}{l}\text { Estimated lost productivity } \\
\text { yesterday }([Y+Z] / X)(\%)\end{array}$ & 60.5 & 30.4 & 69.2 & 33.3 & 50.7 \\
\hline
\end{tabular}

$\mathrm{MOH}$ medication-overuse headache

burden, which can be assessed regardless of headache type, and its impact at population level $(3.0 \%$ loss of productivity).

This is the fourth published analysis of HY, following those in China [15], eight countries of Europe [17] and Russia [16]. Comparison with Russia is of most interest because headache prevalences in China are much lower [23] and the data from the Eurolight study were not entirely population-based [24]. In Russia, $14.5 \%$ of participants, also aged 18-65 years, reported HY [16]. This much higher proportion (14.5\% versus $5.9 \%$ ) reflected a lower prevalence of migraine in Russia than in Karnataka $(20.3 \%$ versus $25.2 \%)$ and lower prevalence of TTH (30.9 \% versus $35.1 \%$ ), but a much higher prevalence of headache on $\geq 15$ days/month $(10.5 \%$ versus $3.0 \%)[5,25]$. As noted above, high-frequency headache has a dominant influence on HY.

The mean duration of HY of $7 \mathrm{~h}$ in Karnataka compared with $6 \mathrm{~h}$ in Russia, where there was a higher rate of medication use [16]. While we calculated that $1.7 \%$ of the adult population in Karnataka had headache at any moment in time, in Russia, the proportion was much higher (3.6 \% [16])-more than double. However, China reported a very similar $1.8 \%$ [15]. In terms of pain intensity, the mean of 2.0 on a scale of $1-3$ was much the same as the 2.1 reported in Russia [16], and expected to be disabling. The consequences were seen in the per-person lost productivity of $50.7 \%$ and population-level lost productivity of $3.0 \%$. Per-person lost productivity was lower in Russia, at $39.8 \%$, but, with the higher prevalence, population-level lost productivity attributed to HY was quite a lot higher than in Karnataka, at $5.8 \%$ [16]. Notwithstanding this, the $3.0 \%$ losses in Karnataka represent a major socioeconomic drain.

While 5.9 \% of participants reported HY in Karnataka, this number was closely corroborated by the predicted 1 -day prevalence of headache of $5.6 \%$. In all three earlier published studies of HY, similar internal consistency has been seen between reported HY and prediction of 1day headache prevalence based on estimated 1-year prevalence and recalled frequency during the preceding 3 months [15-17]. What was underestimated in recall over 3 months was the productivity loss, which was calculated at population level in Karnataka at only $1.8 \%$ [18])-seemingly a major error. Similar underestimates in recall were reported in Russia [16].

The crucial question, which has been raised before [5, 18], is whether and to what extent these findings are representative of this culturally and environmentally very diverse country and can, therefore, be extrapolated to all India. The truth is that we do not know: we have drawn attention to the need for at least one further similar study in the country, perhaps in the north, seeking corroboration [18]. In the meantime, we reiterate that these are the best data available for the country. If it is the case that $1.7 \%$ of the Indian adult population (approximately 14 million people), or anything in the region of this number, have disabling headache at every moment, this signals a pressing need to do something about it: cost-effective treatments are available [26]. At the least, the further study we have called for should be commissioned urgently. The donothing option is not cost-free: it incurs the estimated $3.0 \%$ lost productivity, which is likely to be reflected in a correspondingly diminished gross domestic product. It should be noted that this survey was conducted among the population aged 18-65 years: ie, those of working age, among whom productivity losses have most impact on the population economy. The cost is therefore huge.

The strengths of our study were several. Enquiry into headache yesterday is a powerful approach to populationbased burden-of-headache studies, virtually eliminating recall error and any bias that may follow from this [9]. Our sample was large and representative, and the nonparticipation proportion was low $(7.4 \%)[5,19]$. Through cold-calling and face-to-face interviews, we avoided the previously described bias of "headache today" [17]: participants with headache on the day they receive a questionnaire by post may defer responding until the first day of headache-freedom, and then spuriously report HY.

\section{Conclusions}

Using $\mathrm{HY}$ as a method of enquiry free from recall error, we have confirmed a very high level of headacheattributed burden in Karnataka. Previous estimates based 
on 3-month recall [18] may even have been too low. Until another study is done in the country, these are the best data for all India. We repeat our call for action, reiterating that correct action will ultimately almost certainly be cost-saving [27].

\section{Acknowledgements}

This work was financially supported by Lifting The Burden, a UK-registered non-governmental organization conducting the Global Campaign against Headache in official relations with the World Health Organization. We are grateful to Mr Remees Raj, Data Manager, Centre for Public Health, NIMHANS, for support in data analysis.

\section{Authors' contributions}

TJS and LJS conceived the study and drafted the protocol and study questionnaire, which were subsequently refined in discussion with GG. GG oversaw the study in Karnataka and took responsibility for quality control. GBK and GNR took charge of conduct of the study in the field, with GG acting as principal investigator. GNR took responsibility for the statistical analysis. TJS drafted the manuscript. All authors reviewed and approved the final version.

\section{Competing interests}

TJS and LJS are directors and trustees of Lifting The Burden.

\section{Ethics approval and consent to participate}

The institutional ethics committee of the National Institute for Mental Health and Neuro-Science (NIMHANS), Bengaluru, approved the study protocol. Informed consent was obtained from all participants.

\section{Author details}

'Department of Neuroscience, Norwegian University of Science and Technology (NTNU), Edvard Griegs Gate, Trondheim NO-7491, Norway. ${ }^{2}$ Division of Brain Sciences, Imperial College London, London, UK. ${ }^{3}$ Department of Epidemiology, National Institute of Mental Health and Neuro Sciences (NIMHANS), Bengaluru, India. ${ }^{4}$ Department of Neurology, National Institute of Mental Health and Neuro Sciences (NIMHANS), Bengaluru, India. ${ }^{5}$ Norwegian Advisory Unit on Headache, Nevrosenteret Øst, St Olavs University Hospital, Trondheim, Norway.

Received: 29 June 2016 Accepted: 17 August 2016

Published online: 25 August 2016

\section{References}

1. Vos T, Barber RM, Bell B, Bertozzi-Villa A, Biryukov S, Bolliger I, Charlson F, Davis A, Degenhardt L, Dicker D, Duan L, Erskine H, Feigin VL, Ferrari AJ, Fitzmaurice C, Fleming T, Graetz N, Guinovart C, Haagsma J, Hansen GM Hanson SW, Heuton KR, Higashi H, Kassebaum N, Kyu H, Laurie E, Liang X, Lofgren K, Lozano R, Maclntyre MF, Moradi-Lakeh M, Naghavi M, Nguyen G, Odell S, Ortblad K et al (2015) Global, regional, and national incidence, prevalence, and years lived with disability for 301 acute and chronic diseases and injuries in 188 countries, 1990-2013: a systematic analysis for the Global Burden of Disease Study 2013. Lancet 386:743-800

2. Steiner TJ, Birbeck GL, Jensen RH, Katsarava Z, Stovner LJ, Martelletti $P$ (2015) Headache disorders are third cause of disability worldwide. J Headache Pain 16:58

3. Vos T, Flaxman AD, Naghavi M, Lozano R, Michaud C, Ezzati M, Shibuya K, Salomon JA, Abdalla S, Aboyans V, Abraham J, Ackerman I, Aggarwal R, Ahn SY, Ali MK, Alvarado M, Anderson HR, Anderson LM, Andrews KG, Atkinson C, Baddour LM, Bahalim AN, Barker-Collo S, Barrero LH, Bartels DH, Basáñez MG, Baxter A, Bell ML, Benjamin EJ, Bennett D, Bernabé E, Bhalla K, Bhandari B, Bikbov B, Bin Abdulhak A et al (2012) Years lived with disability (YLDs) for 1160 sequelae of 289 diseases and injuries 1990-2010: a systematic analysis for the Global Burden of Disease Study 2010. Lancet 380:2163-2196

4. Katsarava Z, Dzagnidze A, Kukava M, Mirvelashvili E, Djibuti M, Janelidze M, Jensen R, Stovner LJ, Steiner TJ (2009) Primary headache disorders in the Republic of Georgia: prevalence and risk factors. Neurology 73:1796-1803

5. Kulkarni GB, Rao GN, Gururaj G, Stovner LJ, Steiner TJ (2015) Headache disorders and public ill-health in India: prevalence estimates in Karnataka State. J Headache Pain 16:67
6. Manandhar K, Risal A, Steiner TJ, Holen A, Linde M (2015) The prevalence of primary headache disorders in Nepal: a nationwide population-based study. $J$ Headache Pain 16:95

7. Mbewe E, Zairemthiama P, Yeh HH, Paul R, Birbeck GL, Steiner TJ (2015) The epidemiology of primary headache disorders in Zambia: a population-based door-to-door survey. J Headache Pain 16:30

8. Stovner LJ, Al Jumah M, Birbeck GL, Gururaj G, Jensen R, Katsarava Z, Queiroz LP, Scher Al, Tekle-Haimanot R, Wang SJ, Steiner TJ (2014) The methodology of population surveys of headache prevalence, burden and cost: Principles and recommendations from the Global Campaign against Headache. J Headache Pain 15:5

9. Steiner TJ, Gururaj G, Andrée C, Katsarava Z, Ayzenberg I, Yu S-Y, Al Jumah M, Tekle-Haimanot R, Birbeck GL, Herekar A, Linde M, Mbewe $E$, Manandhar K, Risal A, Jensen R, Queiroz LP, Scher Al, Wang SJ, Stovner LJ (2014) Diagnosis, prevalence estimation and burden measurement in population surveys of headache: presenting the HARDSHIP questionnaire. J Headache Pain 15:3

10. Steiner TJ (2004) Lifting the burden: the global campaign against headache Lancet Neurol 3:204-205

11. Steiner TJ (2005) Lifting The Burden: The global campaign to reduce the burden of headache worldwide. J Headache Pain 6:373-377

12. Steiner TJ, Birbeck GL, Jensen R, Katsarava Z, Martelletti P, Stovner LJ (2010) Lifting The Burden: the first 7 years. J Headache Pain 11:451-455

13. Steiner TJ, Stovner LJ, Dua T, Birbeck GL, Jensen R, Katsarava Z Martelletti P, Saxena S (2011) Time to act on headache disorders. J Headache Pain 12:501-503

14. Steiner TJ (2007) The HALT and HART indices. J Headache Pain 8(Suppl 1):S22-S25

15. Yu S, He M, Liu R, Feng J, Qiao X, Yang X, Cao X, Zhao G, Fang Y, Steiner TJ (2013) Headache yesterday in China: a new approach to estimating the burden of headache, applied in a general-population survey in China. Cephalalgia 33:1211-1217

16. Ayzenberg I, Katsarava Z, Sborowski A, Obermann M, Chernysh M, Osipova V, Tabeeva G, Steiner TJ (2015) Headache yesterday in Russia: its prevalence and impact, and their application in estimating the national burden attributable to headache disorders. J Headache Pain 16:7

17. Andrée C, Steiner TJ, Barré J, Katsarava Z, Lainez JM, Lampl C, Lantéri-Minet M, Rastenyte D, Ruiz de la Torre E, Tassorelli C, Stovner LJ (2014) Headache yesterday in Europe. J Headache Pain 15:33

18. Rao GN, Kulkarni GB, Gururaj G, Stovner LJ, Steiner TJ (2015) The burden attributable to headache disorders in India: estimates from a communitybased study in Karnataka State. J Headache Pain 16:94

19. Rao GN, Kulkarni GB, Gururaj G, Rajesh K, Subbakrishna DK, Steiner TJ, Stovner LJ (2012) The burden of headache disorders in India: methodology and questionnaire validation for a community-based survey in Karnataka State. J Headache Pain 13:543-550

20. Peters M, Bertolote JM, Houchin C, Kandoura T, Steiner TJ (2007) Translation protocol for hybrid documents. J Headache Pain 8(Suppl 1):S40-\$47

21. Headache Classification Subcommittee of the International Headache Society (2004) The International Classification of Headache Disorders: 2nd edition. Cephalalgia 24(Suppl 1):9-160

22. Dean AG, Arner TG, Sunki GG, Friedman R, Lantinga M, Sangam S, Zubieta JC, Sullivan KM, Brendel KA, Gao Z, Fontaine N, Shu M, Fuller G, Smith DC, Nitschke DA, Fagan RF (2007) Epi Info ${ }^{\mathrm{TM}}$, a database and statistics program for public health professionals, version 3.5.1. Centers for Disease Control and Prevention, Atlanta

23. Yu S, Liu R, Zhao G, Yang X, Qiao X, Feng J, Fang Y, Cao X, He M, Steiner T (2012) The prevalence and burden of primary headaches in China: a population-based door-to-door survey. Headache 52:582-591

24. Andrée C, Stovner LJ, Steiner TJ, Barré J, Katsarava Z, Lainez JM, Lair M-L, Lanteri-Minet M, Mick G, Rastenyte D, Ruiz de la Torre E, Tassorelli C, Vriezen P, Lampl C (2011) The Eurolight project: the impact of primary headache disorders in Europe. Description of methods. J Headache Pain 12:541-549

25. Ayzenberg I, Katsarava Z, Sborowski A, Chernysh M, Osipova V, Tabeeva G, Yakhno N, Steiner TJ (2012) The prevalence of primary headache disorders in Russia: a countrywide survey. Cephalalgia 32:373-381

26. Linde M, Steiner TJ, Chisholm D (2015) Cost-effectiveness analysis of interventions for migraine in four low- and middle-income countries. J Headache Pain 16:15

27. World Health Organization, Lifting The Burden (2011) Atlas of headache disorders and resources in the world 2011. WHO, Geneva 